\title{
Effect of Fertigation, Irrigation and Mulching on Growth, Flowering and Yield Parameters in African Marigold
}

\author{
K. Raja Babu ${ }^{*}$, H.P. Sumangala ${ }^{2}$, T.R. Rupa $^{3}$, Sangama ${ }^{4}$ and Sujatha A. Nair ${ }^{4}$ \\ ${ }^{1}$ College of Horticulture, Dr. Y.S.R Horticultural University, V.R. Gudem-534101, \\ Andhra Pradesh, India \\ ${ }^{2}$ (Floriculture and Medicinal crops), ICAR-IIHR, Hesaraghatta-560089, Bengaluru, \\ Karnataka, India \\ ${ }^{3}$ (Soil Science and Agricultural Technology), ICAR-IIHR, Hesaraghatta-560089, Bengaluru, \\ Karnataka, India \\ ${ }^{4}$ (Horticulture), ICAR-IIHR, Hesaraghatta-560089, Bengaluru, Karnataka, India \\ *Corresponding author
}

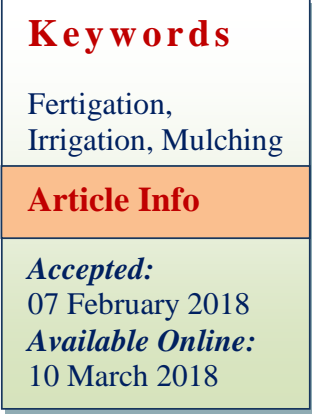

\section{A B S T R A C T}

The experiment was conducted with three levels of Irrigation treatments viz., 1.0, 0.8 and 0.6 ER and six levels of fertigation and mulching treatments viz., fertigation of water soluble fertilizers (WSF) @ 100\% recommended dose of fertilizers (RDF) with mulching, fertigation of WSF @ 75\% RDF with mulching, fertigation of WSF @ $100 \%$ RDF without mulching, fertigation of WSF @ 100\% RDF without mulching, soil application of normal fertilizers @ 100\% RDF with mulching and soil application of normal fertilizers @ 100\% RDF without mulching. Among irrigation treatments, 1.0 Evaporation Replenishment (ER) recorded highest values for parameters like plant height $(47.98 \mathrm{~cm})$, primary branches $(7.73)$, plant spread $(81.17 \mathrm{~cm})$, dry matter production $(58.87 \mathrm{~g})$, days to flower bud initiation (34.05 days), duration of flowering (77.74 days), flower diameter $(5.57 \mathrm{~cm})$, shelf life (3.56 days), individual flower weight $(10.47 \mathrm{~g})$, Flower yield per plant $(748.54 \mathrm{~g})$, Flower yield per plot $(44.64 \mathrm{~kg})$ and Flower yield per hectare (13.40 tn). 0.8 Evaporation Replenishment (ER) recorded highest values in case of days to $50 \%$ flowering (60.10), Number of flowers per plant (84.83). Among fertigation and mulching treatments, Fertigation with water soluble fertilizers@100\% RDF and polyethylene mulching recorded highest values for parameters like plant height $(51.10 \mathrm{~cm})$, primary branches $(8.05)$, plant spread $(89.54$ $\mathrm{cm})$, stem girth $(10.07 \mathrm{~cm})$, dry matter production $(66.21 \mathrm{~g})$, days to flower bud initiation (35.05 days), duration of flowering ( 77.73 days), flower diameter $(5.75 \mathrm{~cm})$, shelf life (4.08 days), Number of flowers per plant (88.08), individual flower weight $(11.04 \mathrm{~g})$, flower yield per plant $(770.25 \mathrm{~g})$, flower yield per plot $(46.72 \mathrm{~kg})$ and Flower yield per hectare $(14.01 \mathrm{tn})$. Among interactions 1.0ER+ Fertigation with water soluble fertilizers @ 100\% RDF and polyethylene mulching recorded highest values for growth, flowering and yield parameters. 


\section{Introduction}

African marigold (Tagetes erecta L.) belongs to family Asteraceae. It is an important traditional flower crop most commonly cultivated and used flower in urban and rural India. Marigold flowers are extensively used for making garlands, beautification, religious offerings, social functions and other purposes such as pigment and oil extraction and therapeutic uses. Apart from these uses, marigold is widely grown in gardens and pots for display purpose. Marigold gains popularity among garden lovers and loose flower dealers on account of its free flowering habit, short duration, attractive colour, form and keeping quality. Fertigation is a method of fertilization in which nutrients along with water are applied directly to the root zone of the plant in small but frequent quantities through the drippers (Battilani, 1997 and Raina, 2000). The timing and rate of irrigation should be adjusted to correspond to the plants water demand with an ambition to produce good yields of prime quality. Compared to furrow irrigation, drip irrigation provides better water use efficiency. Mulching increases the soil temperature and moisture; control the weeds besides improving the chemical and physical properties of soil thereby improving the productivity of the crop. In the era of declining resources there is need to standardize precision farming technologies for farmers with the aim to enhance the productivity and to reduce water foot print per unit of crop produce. Therefore, the present study aimed to examine the effect of fertigation, irrigation and mulching on the productivity of marigold

\section{Materials and Methods}

The experiment was conducted during kharif season of 2016, at the Division of Floriculture and medicinal crops, ICAR-Indian Institute of Horticultural Research (ICAR-IIHR),
Bengaluru. The experiment laid out on split plot design by keeping Irrigation as a main plot viz., 1.0, 0.8 and 0.6 ER as a main plots and fertigation and mulching given as sub plot treatments viz., fertigation of WSF @ 100\% RDF with polyethylene mulching, fertigation of WSF @ 75\% RDF with polyethylene mulching, fertigation of WSF @ 100\% RDF without polyethylene mulching, fertigation of WSF @ 100\% RDF without polyethylene mulching, soil application of normal fertilizers @ 100\% RDF with polyethylene mulching and soil application of normal fertilizers @ $100 \%$ RDF without polyethylene mulching. The composite soil of the experimental plot was slightly alkaline $(7.35 \mathrm{pH})$ in soil reaction and non-saline $\left(0.26 \mathrm{~d} \mathrm{Sm}^{-1}\right)$ in nature. The soil was low in organic carbon $(0.41 \%)$ content. Organic manure i.e., farmyard manure $(20 \mathrm{t})$ were applied in the pre marked rows as per the treatments five days earlier to transplanting. The recommended dose of fertilizers (NPK @ 100:75:75 kg ha ${ }^{-1}$ )was applied based on treatments (through fertigation) in the form of water soluble fertilizers (Urea, 19:19:19).

The fertigation was given at weekly intervals twenty days after transplanting up to 70 days. Fertigation was given by using gutter spray. For direct soil application treatments Normal fertilizers like Urea, DAP and MOP (585, 1265 and $337 \mathrm{~g}$, respectively) were applied at the time of bed preparation.

Scheduling of irrigation was started 20 days after transplanting till final harvest. Irrigation was scheduled in once in two days. Previous two days open pan evaporimeter was considered for scheduling that irrigation. The Irrigation schedule was calculated by using below formula.

Bed Size X pan evaporation rate Evaporation Replenishment $(\mathrm{ER})=$ Discharge capacity of drip per minute 
Observations on vegetative parameters like plant height, number of primary and secondary branches per plant, plant spread, stem girth and dry matter production, floral characters like days to flower bud initiation, days to first flower opening, days to $50 \%$ flowering, days to $100 \%$ flowering, duration of flowering, flower diameter and shelf life, yield parameters like Number of buds per plant, Number of flowers per plant, Average weight of 10 fresh flowers (g), individual flower weight (g), Flower yield per plant $(\mathrm{g})$, Flower yield per plot $(\mathrm{Kg})$, Flower yield per hectare (tn) were recorded.

\section{Results and Discussion}

\section{Irrigation}

The vegetative growth, flowering and yield were significantly influenced by different irrigation levels. The results obtained with the irrigation treatments, 1.0 Evaporation Replenishment (ER) recorded highest values for parameters like plant height $(47.98 \mathrm{~cm})$, primary branches (7.73), secondary branches per plant (13.11), plant spread $(81.17 \mathrm{~cm})$, stem girth $(8.64 \mathrm{~cm})$, dry matter production $(58.87 \mathrm{~g})$, days to flower bud initiation (34.05 days), days to first flower opening (40.07 days), duration of flowering (77.74 days), flower diameter $(5.57 \mathrm{~cm})$, shelf life $(3.56$ days), Number of buds per plant (88.66), Average weight of 10 fresh flowers (106.46 $\mathrm{g}$ ), individual flower weight (10.47 g), Flower yield per plant (748.54 g), Flower yield per plot $(44.64 \mathrm{~kg})$ and Flower yield per hectare (13.40 tn). 0.8 Evaporation Replenishment (ER) recorded highest values in case of days to $50 \%$ flowering (60.10), days to $100 \%$ flowering (69.50), Number of flowers per plant (84.83). Irrigation at 1.0 ER shows better among all vegetative parameters due to progressive increase in irrigation levels recorded significant improvement of plant. Adequate supply of water leads to growth and development of plant, continuing cell division and cell enlargement, progressive initiation of tissue and primordial. Similar results were found in Khanam and Patra (2015) in Gladiolus, Khalid et al., (2015) in Zinnia, Ayyanna et al., (2014). Among all floral parameters application high amount of irrigation recorded highest due to the increased dry matter production at successive stages with increased water availability might have led to greater availability of photosynthates towards formation of reproductive structure of floral primordial and their growth results in better floral initiation and development.

\section{Fertigation and mulching}

The Fertigation and mulching had significant effect on the growth, flowering and yield parameters. The results among fertigation and mulching treatments, Fertigation with water soluble fertilizers @ 100 \% $\quad$ RDF and polyethylene mulching recorded highest values for parameters like plant height (51.10 $\mathrm{cm}$ ), primary branches (8.05), secondary branches per plant (14.08), plant spread (89.54 $\mathrm{cm})$, stem girth $(10.07 \mathrm{~cm})$, dry matter production $(66.21 \mathrm{~g})$, days to flower bud initiation (35.05 days), days to first flower opening (40.98 days), days to $50 \%$ flowering (57.52 days), days to $100 \%$ flowering (66.34 days), duration of flowering (77.73 days), flower diameter $(5.75 \mathrm{~cm})$, shelf life $(4.08$ days), number of buds per plant (92.74), Number of flowers per plant (88.08), average weight of 10 fresh flowers (110.51), individual flower weight (11.04), flower yield per plant $(770.25 \mathrm{~g})$, flower yield per plot $(46.72 \mathrm{~kg})$ and Flower yield per hectare (14.01 tn).The increase in vegetative parameters through fertigation was obtained due to availability of the WSF at rhizosphere with reduced nutrient losses by leaching and efficient use of nutrients through fertigation (Paul et al., 1992) (Table 1-3). 
Table.1 Effect of fertigation, irrigation and mulching on growth, flowering and yield parameters in African marigold

\begin{tabular}{|c|c|c|c|c|c|c|}
\hline \multirow[t]{2}{*}{ Treatment } & \multirow{2}{*}{$\begin{array}{c}\text { Plant } \\
\text { height } \\
\text { (cm) }\end{array}$} & \multicolumn{2}{|c|}{ Number of } & \multirow{2}{*}{$\begin{array}{l}\text { Plant } \\
\text { Spread } \\
\text { (cm) }\end{array}$} & \multirow{2}{*}{$\begin{array}{l}\text { Stem Girth } \\
(\mathrm{cm})\end{array}$} & \multirow{2}{*}{$\begin{array}{l}\text { Dry matter } \\
\text { production (g) }\end{array}$} \\
\hline & & $\begin{array}{l}\text { Primary } \\
\text { branches }\end{array}$ & $\begin{array}{c}\text { Secondary } \\
\text { branches }\end{array}$ & & & \\
\hline \multicolumn{7}{|l|}{ Irrigation } \\
\hline$I_{1}(1.0 \mathrm{ER})$ & 47.98 & 7.73 & 13.11 & 81.17 & 8.64 & 58.87 \\
\hline $\mathrm{I}_{2}(0.8 \mathrm{ER})$ & 46.73 & 7.42 & 12.60 & 79.66 & 8.39 & 54.65 \\
\hline $\mathrm{I}_{3}(\mathbf{0 . 6 E R})$ & 39.23 & 7.24 & 11.42 & 69.87 & 7.49 & 44.54 \\
\hline${ }^{\mathrm{CD}} 0.05$ & 0.84 & 0.07 & 0.96 & 6.37 & 0.10 & 1.06 \\
\hline \multicolumn{7}{|c|}{$\begin{array}{l}\text { Fertigation and } \\
\text { Muching }\end{array}$} \\
\hline $\mathbf{S}_{1}$ & 51.10 & 8.05 & 14.08 & 89.54 & 10.07 & 66.21 \\
\hline$S_{2}$ & 48.84 & 7.89 & 13.26 & 85.24 & 9.11 & 63.15 \\
\hline $\mathbf{S}_{3}$ & 45.98 & 7.30 & 12.85 & 80.66 & 8.14 & 54.03 \\
\hline $\mathrm{S}_{4}$ & 44.35 & 7.19 & 12.65 & 76.95 & 7.76 & 50.36 \\
\hline$S_{5}$ & 39.14 & 7.17 & 11.92 & 66.49 & 7.30 & 40.53 \\
\hline$S_{6}$ & 38.48 & 7.20 & 9.49 & 62.52 & 6.66 & 41.83 \\
\hline $\mathrm{CD}_{0.05}$ & 1.18 & 0.08 & 0.49 & 5.18 & 0.33 & 2.35 \\
\hline \multicolumn{7}{|l|}{ Interactions } \\
\hline $\mathrm{I}_{1} \times \mathrm{S}_{1}$ & 54.57 & 8.80 & 14.64 & 98.57 & 10.87 & 74.00 \\
\hline$I_{1} X S_{2}$ & 53.36 & 8.60 & 14.43 & 93.08 & 9.58 & 72.85 \\
\hline$I_{1} X S_{3}$ & 49.65 & 7.55 & 13.50 & 84.15 & 8.37 & 65.30 \\
\hline $\mathbf{I}_{1} \mathbf{X ~ S _ { 4 }}$ & 47.07 & 7.50 & 13.11 & 81.65 & 8.58 & 58.90 \\
\hline$I_{1} X S_{5}$ & 42.43 & 7.36 & 12.29 & 67.30 & 7.50 & 36.95 \\
\hline$I_{1} X S_{6}$ & 40.79 & 6.60 & 10.70 & 62.68 & 6.97 & 45.25 \\
\hline $\mathbf{I}_{2} \mathbf{X ~ S} \mathbf{S}_{1}$ & 54.29 & 8.10 & 14.48 & 92.14 & 10.58 & 73.95 \\
\hline $\mathbf{I}_{\mathbf{2}} \mathbf{X} \mathbf{S}_{\mathbf{2}}$ & 50.36 & 7.95 & 13.22 & 87.79 & 9.71 & 70.10 \\
\hline $\mathbf{I}_{\mathbf{2}} \mathbf{X ~ S _ { 3 }}$ & 49.64 & 7.40 & 13.07 & 84.50 & 8.5 & 51.25 \\
\hline $\mathrm{I}_{2} \mathrm{XS} \mathrm{S}_{4}$ & 48.57 & 7.33 & 12.93 & 80.36 & 7.41 & 47.85 \\
\hline $\mathbf{I}_{2} \mathbf{X} \mathbf{S}_{5}$ & 39.00 & 7.05 & 12.45 & 68.78 & 7.3 & 42.75 \\
\hline $\mathbf{I}_{2} \mathbf{X ~ S _ { 6 }}$ & 38.55 & 6.73 & 9.50 & 64.40 & 6.86 & 42.00 \\
\hline $\mathbf{I}_{\mathbf{3}} \mathbf{X} \mathbf{S}_{1}$ & 44.43 & 7.25 & 13.14 & 77.93 & 8.76 & 50.70 \\
\hline $\mathbf{I}_{3} X \mathbf{S}_{2}$ & 42.79 & 7.14 & 12.14 & 74.86 & 8.06 & 46.50 \\
\hline$I_{3} X S_{3}$ & 38.65 & 6.95 & 12.00 & 73.36 & 7.56 & 45.55 \\
\hline$I_{3} X S_{4}$ & 37.40 & 6.75 & 11.93 & 68.86 & 7.3 & 44.35 \\
\hline$I_{3} X S_{5}$ & 36.00 & 7.10 & 11.04 & 63.35 & 7.1 & 41.90 \\
\hline $\mathbf{I}_{3} \times \mathbf{S}_{6}$ & 36.10 & 6.52 & 8.28 & 60.90 & 6.16 & 38.25 \\
\hline${ }^{\mathrm{CD}} 0.05$ & 2.12 & 0.15 & NS & NS & 0.58 & 4.13 \\
\hline
\end{tabular}

$\mathrm{S}_{1}$ : Fertigation of WSF@ 100\% RDF with Mulching; $\mathrm{S}_{2}$ : Fertigation of WSF @ 75\% RDF with Mulching

$\mathrm{S}_{3}$ : Fertigation of WSF @ 100\% RDF without Mulching; $\mathrm{S}_{4}$ : Fertigation of WSF @ 75\% RDF without Mulching $\mathrm{S}_{5}$ : Soil application of normal fertilizers @ 100\% RDF with Mulching; $\mathrm{S}_{6}$ : Soil application of normal fertilizers @ $100 \%$ RDF without Mulching 
Table.2 Effect of fertigation, irrigation and mulching on growth, flowering and yield parameters in African marigold

\begin{tabular}{|c|c|c|c|c|c|c|c|}
\hline \multirow[t]{2}{*}{ Treatment } & \multicolumn{4}{|c|}{ Days taken for } & \multirow{2}{*}{$\begin{array}{c}\text { Duration } \\
\text { of } \\
\text { flowering }\end{array}$} & \multicolumn{2}{|c|}{ Number of } \\
\hline & $\begin{array}{c}\text { First flower } \\
\text { bud } \\
\text { initiation }\end{array}$ & $\begin{array}{c}\text { First } \\
\text { flower } \\
\text { opening }\end{array}$ & $\begin{array}{c}\mathbf{5 0 \%} \\
\text { Flowering }\end{array}$ & $\begin{array}{c}100 \% \\
\text { Flowering }\end{array}$ & & $\begin{array}{l}\text { Buds } \\
\text { per } \\
\text { plant }\end{array}$ & $\begin{array}{c}\text { Flowers } \\
\text { per plant }\end{array}$ \\
\hline \multicolumn{8}{|l|}{ Irrigation } \\
\hline $\mathrm{I}_{1}(1.0 \mathrm{ER})$ & 34.05 & 40.07 & 60.10 & 69.50 & 77.74 & 88.66 & 84.83 \\
\hline $\mathrm{I}_{2}(0.8 \mathrm{ER})$ & 35.50 & 42.58 & 60.01 & 67.42 & 73.21 & 88.40 & 84.90 \\
\hline $\mathrm{I}_{3}(0.6 \mathrm{ER})$ & 41.38 & 47.97 & 63.08 & 69.67 & 69.31 & 68.45 & 65.45 \\
\hline${ }^{\mathrm{CD}} 0.05$ & 0.79 & 1.26 & 0.54 & 0.40 & 1.31 & 3.63 & 3.15 \\
\hline \multicolumn{8}{|c|}{$\begin{array}{l}\text { Fertigation and } \\
\text { Muching }\end{array}$} \\
\hline $\mathrm{S}_{1}$ & 35.05 & 40.98 & 57.52 & 66.34 & 77.73 & 92.74 & 88.08 \\
\hline $\mathbf{S}_{2}$ & 35.56 & 42.39 & 59.50 & 68.34 & 76.10 & 88.73 & 84.73 \\
\hline$\overline{S_{3}}$ & 35.21 & 43.43 & 59.34 & 67.01 & 74.13 & 81.28 & 78.28 \\
\hline $\mathbf{S}_{4}$ & 37.25 & 43.05 & 61.68 & 69.33 & 71.46 & 78.35 & 75.35 \\
\hline$\overline{S_{5}}$ & 38.42 & 45.07 & 63.52 & 70.00 & 72.21 & 80.73 & 76.73 \\
\hline$\overline{S_{6}}$ & 40.38 & 46.31 & 64.83 & 72.16 & 68.90 & 69.18 & 67.18 \\
\hline${ }^{\mathrm{CD}} 0.05$ & 0.97 & 0.31 & 0.53 & 0.52 & 0.48 & 1.08 & 0.97 \\
\hline \multicolumn{8}{|l|}{ Interactions } \\
\hline $\mathrm{I}_{1} \mathbf{X ~ S _ { 1 }}$ & 31.50 & 36.50 & 49.00 & 62.50 & 85.30 & 97.78 & 92.78 \\
\hline $\mathbf{I}_{1} \mathbf{X ~ S} \mathbf{S}_{2}$ & 32.40 & 38.50 & 56.50 & 69.50 & 81.76 & 96.15 & 92.15 \\
\hline $\mathbf{I}_{\mathbf{1}} \mathbf{X} \mathbf{S}_{\mathbf{3}}$ & 31.96 & 41.00 & 61.03 & 63.00 & 78.50 & 88.50 & 83.50 \\
\hline $\begin{array}{l}\mathbf{I} X \mathbf{S}_{4} \\
\end{array}$ & 33.60 & 37.43 & 63.03 & 74.00 & 74.86 & 84.90 & 81.91 \\
\hline $\mathbf{I}_{1} \mathbf{X ~ S} \mathbf{S}_{5}$ & 37.10 & 42.00 & 65.03 & 72.50 & 73.50 & 89.06 & 85.06 \\
\hline $\mathbf{I}_{1} \mathbf{X} \mathbf{S}_{6}$ & 37.75 & 45.00 & 66.00 & 65.50 & 72.50 & 75.58 & 73.58 \\
\hline $\mathbf{I}_{2} \mathbf{X} \mathbf{S}_{1}$ & 31.65 & 39.32 & 58.53 & 68.53 & 76.43 & 99.92 & 93.92 \\
\hline $\mathbf{I}_{2} \mathbf{X} \mathbf{S}_{2}$ & 33.30 & 40.73 & 59.50 & 70.50 & 76.00 & 95.71 & 91.71 \\
\hline $\mathbf{I}_{\mathbf{2}} \mathbf{X} \mathbf{S}_{\mathbf{3}}$ & 33.30 & 40.98 & 58.00 & 64.50 & 74.50 & 87.78 & 85.79 \\
\hline $\mathbf{I}_{2} \mathbf{X ~ S _ { 4 }}$ & 37.20 & 43.74 & 58.53 & 67.00 & 70.87 & 86.78 & 83.78 \\
\hline $\mathbf{I}_{2} \mathbf{X} \mathbf{S}_{5}$ & 36.65 & 45.23 & 61.00 & 67.50 & 71.12 & 86.06 & 82.06 \\
\hline $\mathrm{I}_{2} \mathrm{X} \mathbf{S}_{6}$ & 40.90 & 45.50 & 66.50 & 75.50 & 70.35 & 74.13 & 72.13 \\
\hline $\mathbf{I}_{3} \mathbf{X} \mathbf{S}_{1}$ & 42.00 & 47.12 & 65.00 & 68.00 & 71.46 & 80.54 & 77.54 \\
\hline $\mathbf{I}_{3} \mathbf{X} \mathbf{S}_{2}$ & 41.00 & 47.96 & 62.50 & 65.03 & 70.53 & 74.34 & 70.34 \\
\hline $\mathbf{I}_{\mathbf{3}} \mathbf{X} \mathbf{S}_{\mathbf{3}}$ & 40.35 & 48.32 & 59.00 & 73.50 & 69.35 & 67.56 & 65.56 \\
\hline $\mathbf{I}_{3} \mathbf{X ~ S} \mathbf{S}_{4}$ & 40.95 & 47.99 & 63.50 & 67.00 & 68.67 & 63.38 & 60.38 \\
\hline $\mathbf{I}_{\mathbf{3}} \mathbf{X} \mathbf{S}_{5}$ & 41.50 & 48.00 & 64.50 & 70.00 & 72.00 & 67.08 & 63.08 \\
\hline $\mathrm{I}_{3} \mathrm{X} \mathrm{S_{6 }}$ & 42.50 & 48.44 & 64.00 & 74.50 & 63.86 & 57.83 & 55.83 \\
\hline${ }^{\mathrm{CD}} 0.05$ & 1.71 & 1.34 & 0.99 & 0.91 & 1.02 & 2.36 & 2.17 \\
\hline
\end{tabular}

$\mathrm{S}_{1}$ : Fertigation of WSF@ 100\% RDF with Mulching; $\mathrm{S}_{2}$ : Fertigation of WSF @ 75\% RDF with Mulching

$\mathrm{S}_{3}$ : Fertigation of WSF @ 100\% RDF without Mulching; $\mathrm{S}_{4}$ : Fertigation of WSF @ 75\% RDF without Mulching

$\mathrm{S}_{5}$ : Soil application of normal fertilizers @ 100\% RDF with Mulching; $\mathrm{S}_{6}$ : Soil application of normal fertilizers @ $100 \%$ RDF without Mulching 
Int.J.Curr.Microbiol.App.Sci (2018) 7(3): 685-692

Table.3 Effect of fertigation, irrigation and mulching on growth, flowering and yield parameters in African marigold

\begin{tabular}{|c|c|c|c|c|c|c|c|}
\hline Treatment & $\begin{array}{c}\text { Flower } \\
\text { diameter } \\
(\mathrm{cm})\end{array}$ & $\begin{array}{l}\text { Shelf } \\
\text { life } \\
\text { (days) }\end{array}$ & $\begin{array}{l}10 \text { fresh } \\
\text { flower } \\
\text { weight }(\mathrm{g})\end{array}$ & $\begin{array}{l}\text { Individual } \\
\text { flower } \\
\text { weight (g) }\end{array}$ & $\begin{array}{c}\text { Yield/plant } \\
\text { (g) }\end{array}$ & $\begin{array}{l}\text { Yield per } \\
\text { plot (kg) }\end{array}$ & $\begin{array}{l}\text { Yield/Ha } \\
\text { (tn) }\end{array}$ \\
\hline \multicolumn{8}{|l|}{ Irrigation } \\
\hline $\mathrm{I}_{1}(1.0 \mathrm{ER})$ & 5.57 & 3.56 & 106.46 & 10.47 & 748.54 & 44.64 & 13.40 \\
\hline $\mathrm{I}_{2}(0.8 \mathrm{ER})$ & 5.50 & 3.25 & 100.01 & 9.89 & 722.41 & 41.84 & 12.04 \\
\hline $\mathrm{I}_{3}(0.6 \mathrm{ER})$ & 4.47 & 2.69 & 79.98 & 7.97 & 655.16 & 29.78 & 9.10 \\
\hline${ }^{\mathrm{CD}} 0.05$ & 0.28 & 0.13 & 1.36 & 0.32 & 19.78 & 2.47 & 1.96 \\
\hline \multicolumn{8}{|l|}{$\begin{array}{l}\text { Fertigation } \\
\text { and Muching } \\
\end{array}$} \\
\hline $\mathrm{S}_{1}$ & 5.75 & 4.08 & 110.51 & 11.04 & 770.25 & 46.72 & 14.01 \\
\hline$S_{2}$ & 5.37 & 3.33 & 101.82 & 9.91 & 742.84 & 43.75 & 13.06 \\
\hline $\mathbf{S}_{\mathbf{3}}$ & 5.21 & 3.67 & 94.33 & 9.35 & 729.11 & 37.97 & 10.97 \\
\hline$S_{4}$ & 5.07 & 2.68 & 93.41 & 9.32 & 716.54 & 35.17 & 10.46 \\
\hline$S_{5}$ & 4.99 & 3.05 & 91.97 & 8.68 & 653.85 & 38.01 & 10.71 \\
\hline$S_{6}$ & 4.68 & 2.17 & 80.87 & 8.37 & 639.63 & 30.90 & 9.88 \\
\hline${ }^{\mathrm{CD}} 0.05$ & 0.16 & 0.09 & 2.48 & 0.30 & 28.10 & 0.80 & 1.24 \\
\hline \multicolumn{8}{|l|}{ Interactions } \\
\hline $\mathrm{I}_{1} \times \mathrm{S} \mathrm{S}_{1}$ & 6.23 & 4.50 & 123.75 & 12.36 & 805.71 & 53.68 & 16.52 \\
\hline$I_{1} \times S_{2}$ & 5.70 & 4.00 & 111.22 & 10.80 & 781.07 & 50.06 & 15.40 \\
\hline$I_{1} X S_{3}$ & 5.59 & 4.00 & 104.84 & 10.30 & 767.50 & 42.64 & 11.96 \\
\hline $\mathbf{I}_{1} \mathbf{X ~ S} \mathbf{S}_{4}$ & 5.37 & 3.03 & 106.25 & 10.60 & 765.71 & 40.32 & 11.32 \\
\hline $\mathbf{I}_{1} \mathbf{X} \mathbf{S}_{5}$ & 5.28 & 3.33 & 103.43 & 9.50 & 697.71 & 44.32 & 12.97 \\
\hline$I_{1} X S_{6}$ & 5.25 & 2.50 & 89.26 & 9.30 & 673.56 & 36.83 & 12.25 \\
\hline $\mathbf{I}_{\mathbf{2}} \mathbf{X} \mathbf{S}_{1}$ & 6.01 & 4.26 & 117.50 & 11.76 & 800.43 & 53.11 & 15.25 \\
\hline $\mathbf{I}_{2} \mathbf{X} \mathbf{S}_{2}$ & 5.69 & 3.50 & 112.50 & 10.76 & 748.29 & 50.35 & 14.30 \\
\hline$I_{2} X S_{3}$ & 5.50 & 4.00 & 97.92 & 9.76 & 739.99 & 40.58 & 11.50 \\
\hline $\mathbf{I}_{2} \mathbf{X ~ S _ { 4 }}$ & 5.41 & 3.03 & 95.42 & 9.54 & 731.79 & 36.07 & 11.10 \\
\hline$I_{2} \times S_{5}$ & 5.35 & 2.70 & 92.60 & 8.90 & 658.61 & 40.15 & 10.70 \\
\hline $\mathbf{I}_{2} \mathbf{X ~ S _ { 6 }}$ & 5.05 & 2.00 & 84.15 & 8.66 & 655.36 & 30.82 & 9.44 \\
\hline $\mathbf{I}_{3} \mathbf{X} \mathbf{S}_{1}$ & 5.02 & 3.50 & 90.25 & 9.02 & 704.62 & 33.39 & 10.27 \\
\hline $\mathbf{I}_{3} X \mathbf{S}_{2}$ & 4.75 & 2.50 & 81.75 & 8.18 & 699.18 & 30.86 & 9.49 \\
\hline $\mathbf{I}_{3} \mathbf{X ~ S _ { 3 }}$ & 4.55 & 3.03 & 80.25 & 8.02 & 681.65 & 30.70 & 9.44 \\
\hline $\mathbf{I}_{3} \mathrm{X} \mathrm{S}_{4}$ & 4.45 & 2.00 & 78.55 & 7.86 & 650.36 & 29.13 & 8.96 \\
\hline $\mathbf{I}_{\mathbf{3}} \mathbf{X} \mathbf{S}_{\mathbf{5}}$ & 4.35 & 3.13 & 79.83 & 7.66 & 605.22 & 29.58 & 8.46 \\
\hline $\mathbf{I}_{3} \times \mathbf{S}_{6}$ & 3.75 & 2.00 & 69.20 & 7.16 & 589.97 & 25.05 & 7.96 \\
\hline${ }^{\mathrm{CD}} 0.05$ & NS & 0.18 & 4.39 & 0.57 & NS & 1.77 & NS \\
\hline
\end{tabular}

$\mathrm{S}_{1}$ : Fertigation of WSF@ 100\% RDF with Mulching; $\mathrm{S}_{2}$ : Fertigation of WSF @ 75\% RDF with Mulching

$\mathrm{S}_{3}$ : Fertigation of WSF @ 100\% RDF without Mulching; $\mathrm{S}_{4}$ : Fertigation of WSF @ 75\% RDF without Mulching

$\mathrm{S}_{5}$ : Soil application of normal fertilizers @ 100\% RDF with Mulching; $\mathrm{S}_{6}$ : Soil application of normal fertilizers @ $100 \%$ RDF without Mulching 
Similar results were given by Vijay Kumar et al., (2015) in calendula, Kishore (2016) in marigold. The increase in plant height under black polythene mulch might be due to conservation of soil moisture and moderating soil temperature for improved root growth and better absorption of nutrients with better weed control. Similar results were obtained in Solaiman et al., (2008) in China aster. Highest flower diameter due to proper fertigation and high amount of application of fertilizers and due to the role of nitrogen in protein synthesis and augmenting growth. Identical results on gerbera were obtained by Salma et al., (2014) in gerbera, Chouhan et al., (2014) in gladiolus.

\section{Interaction effects}

Interaction effect among the different growth, flowering and yieldparameters plant height, number of primary branches, stem girth, dry matter, days to flower bud initiation, flower opening, days to $50 \%, 100 \%$ flowering, duration of flowering, shelf life, number of buds, flowers per plant, average weight of 10 fresh flowers, individual flower weight, flower yield per plot was significant. Among interaction effects, 1.0 ER + Fertigation with WSF @ 100 \% RDF and polyethylene mulching recorded highest values for parameters like plant height $(53.57 \mathrm{~cm})$, primary branches (8.80), secondary branches per plant (14.64), plant spread $(98.57 \mathrm{~cm})$, stem girth $(10.87 \mathrm{~cm})$, dry matter production (74.00 g), days to flower bud initiation (31.50 days), days to first flower opening (36.50 days), days to $50 \%$ flowering (49.00 days), days to $100 \%$ flowering (62.50 days), duration of flowering (85.30 days), flower diameter $(6.23 \mathrm{~cm})$, shelf life (4.50 days), Average weight of 10 fresh flowers (123.75 g), individual flower weight (12.36 g), Flower yield per plant (805.71 g), Flower yield per plot $(53.38 \mathrm{~kg})$ and Flower yield per hectare $(16.52 \mathrm{tn})$. And combination of $0.8 \mathrm{ER}+$
Fertigation with water soluble fertilizers @ 100 \% RDF and polyethylene mulching recorded highest Number of buds per plant (97.78), Number of flowers per plant (92.78). Higher frequency irrigation created favorable soil water environment for crop growth and resulted in higher yield. It may be stated that the yield increased as a result of keeping the soil water content at required level similar results observed in Tsirogiannis et al., (2010) in gerbera, Kazaz et al., (2010), Aydinsakir et al., (2011) in carnation. Fertigation of higher dose of nutrients which resulted in proper development of photosynthetic system due to which, higher accumulation of dry matter and development of reproductive system occur. These results are in conformation with the findings of Naik (2015) in marigold, Agarwal et al., (2014).

Irrigation with $\quad 1.0 \quad$ Evaporation Replenishment (ER), Fertigation with water soluble fertilizers @ 100 \% RDF and polyethylene mulching are found superior in respect of vegetative, flowering and yield parameters in marigold. They were at par with irrigation level of 0.8 Evaporation Replenishment and fertigation of WSF @ $75 \%$ RDF with polyethylene mulching.

\section{References}

Agarwal, N, Sharma, H.G, Dixit, A, Dubey, P. and Agarwal, S. 2014.Effect of fertigation through water soluble fertilizers on growth, yield and quality of pomegranate. Journal of Agriculture. 9: 13-18.

Aydinsakir, K, Tuzel, I.H. and Buyuktas. D. 2011. The effects of different irrigation levels on flowering and flower quality of carnation (Dianthus caryophllus L.) irrigated by drip irrigation. African Journal of Biotechnology. 10 (66): 1482635. 
Ayyanna, D.S, Basavaraj, S, Polisagowdar, R. and Hiremath. 2014. Evaluation of Surface and Drip irrigation methods for marigold flower (Tagetes erecta L.) under Raichur condition. Acta Biologicalndica, 3 (1): 610-16.

Bittalani, A. 1997. Effect of irrigation and Fertigation on the yield and market quality of nectarines. Acta Horticulture.44: 153-59.

Chouhan, P, Sankar, M.V. and Vijay, R. 2014. Effect of NPK on PhysicoChemical Parameters of Gladiolus (Gladiolus hybridus Hort.) cv. White Prosperity. International Journal of Scientific and Research Publication. 4 (12): 1-5.

Kazaz, S, Yusuf, U, Askin, M.A, Aydinsakir, S.U. and Abdullah, K. 2010.Effects of different irrigation regimes on yield and some quality parameters of carnation. Scientific Research and Essays. 5(19): 2921-30.

Khalid, E, Salah, E.H, Eslam, A.S, Abdallah, E. and Mukhtar Ahmed. 2015. Impacts of fertigation via surface and subsurface drip irrigation on growth rate, yield and flower quality of Zinnia (Zinnia elegans). Bragantia Campinas. 75 (1): 96-107.

Khanam, R. and Patra, S.K. 2015. Effect of gravity Drip Irrigation on Growth, Yield and Water use Efficiency of Gladiolus in Lower Gangetic Plain of West Bengal. International Journal of Ecosystem. 5 (3): 132-37.
Kishore, G.R. 2016.Effect of different levels of nitrogen, phosphorus and potassium on floral characters of African marigold (Tagetes erecta L.) cv. Pusa Narangi Gainda. The Asian Journal of Horticulture. 11 (1): 159-62.

Paul, R.C, Higalu, T. and Simamura, J. 1992.Season and fertilization after the post-harvest flower life of anthurium. Scientia Horticulture. 49: 125-34.

Raina, J.N. 2000. Drip irrigation and fertigation in vegetable crops. Horticulture Technology. Pp. 339-46.

Salma, Z, Pratap, M. and Rao, A.M. 2014. Effect of Different Fertigation Levels on Growth, Quality, Yield and Vase Life of Gerbera Cultivars Grown under Polyhouse Conditions. Annuals of Biology. 30 (1): 186-88.

Solaiman, A.H.M, Kabir, M.H, Jamal Uddin, A.H.M. and Mirza Hasanuzzaman. 2008. Black plastic Mulch on flower production and petal coloration of Aster (Callistephus chinensis). American-Eurasian Journal of Botany. 1(1): 05-08.

Tsirogiannis, I, Katsoulas, N. and Kittas, C. 2010. Effect of Irrigation Scheduling on Gerbera Flower Yield and Quality. Horticulture Sciences. 45 (2): 265-70.

VijayKumar, Pandey, S.K, Singh, V.K, Pushpendra Verty and Sakeel A.S. 2015. Response of nitrogen and phosphorus levels on Calendula (Calendula officinalis L.). Research in Environmental and Life Sciences. 8 (4): 557-60.

\section{How to cite this article:}

Raja Babu, K., H.P. Sumangala, T.R. Rupa, Sangama and Sujatha A. Nair. 2018. Effect of Fertigation, Irrigation and Mulching on Growth, Flowering and Yield Parameters in African Marigold. Int.J.Curr.Microbiol.App.Sci. 7(03): 685-692.

doi: https://doi.org/10.20546/ijcmas.2018.703.080 